\title{
RESEARCH
}

\section{THORACIC DEGENERATIVE DISC PATHOLOGIES IN GERIATRIC PATIENTS}

Turkish Journal of Geriatrics DOI: 10.31086/tigeri.2020.186 2020; 23(4): $482-491$

\section{- Ayşe Serap AKGÜN ${ }^{1}$ (D) \\ - Arzu YAVAŞ DINÇ² (D)}

CORRESPONDANCE

Arzu YAVAŞ DINÇ

Medipol University School of Medicine,

Department of Radiology, Istanbul, Turkey

Phone: +90212912 2525

e-mail: arzudinc0111@gmail.com

Received: Jun 25, 2020

Accepted: Sep 03, 2020

${ }^{1}$ Medipol University School of Medicine, Department of Radiology, İstanbul, Turkey

${ }^{2}$ Medipol University School of Medicine, Department of Physical Therapy and Rehabilitation, Istanbul, Turkey

\section{Abstract}

Introduction: This study investigates the incidence and localization of thoracic degenerative disc pathologies, such as bulging and herniation, in geriatric patients.

Materials and Methods: Between January 2015 and August 2019, a total of 171 patients were retrospectively examined in the study. The patients were admitted for dorsalgia and underwent magnetic resonance imaging (MRI) of the thoracic vertebra. All patients were examined for disc and endplate degeneration, bulging and/or disc herniation, and Schmorl nodes.

Results: Disc bulging/herniation was diagnosed in 534 (26\%) of the overall total of 2052 intervertebral disc levels; 206 (10\%) of these had bulging but no herniation. The location most commonly affected by bulging was T10-11 $(n=46,2.25 \%)$, followed by T11-T12 ( $n=45,2.1 \%)$ Disc herniation was present in $329(15.9 \%)$ of the levels; herniation was most common at T11-12 $(n=51,15.5 \%)$.

Conclusion: The intervention of music therapy was found to have an effect in reducing anxiety and increasing sleep quality of patients. Music therapy can be used as an alternative method of treating the anxiety and increasing the sleep quality of geriatric hematology patients.

Keywords: Geriatric Assessment; Back Pain; Thoracic Vertebrae; Magnetic Resonance Imaging 


\section{INTRODUCTION}

Thoracic disc disease (TDD) is uncommon; it is estimated that less than $1 \%$ of all disc herniations occur in the thoracic region (1). However, several autopsy series have revealed an incidence of $7 \%$ to $15 \%$ $(2,3)$. The majority of thoracic pathologies emerge during the third to fifth decades of life. Disk degeneration is the main cause, but trauma is also considered a major factor (1). The clinical demonstration of a thoracic disc herniation (TDH) depends on its location and morphologic characteristics. This may lead to delayed diagnosis or misdiagnosis of TDH (4). According to previous literature, $75 \%$ of $\mathrm{TDHs}$ occur below $\mathrm{T} 8,3 \%$ of cases occur between $\mathrm{T} 1$ and $\mathrm{T} 2$, and less than $1 \%$ occur between T2 and T3 (5, 6). Cases with TDD usually present with upper back pain or pain radiating to a dermatome. However, the main initial symptom is nonspecific pain, followed by sensory and motor disturbances. Symptoms of spinal cord compression are observed in most patients with TDD at the time of diagnosis (7). Multiple imaging techniques have been used to diagnose this condition; however, as for most vertebral diseases, magnetic resonance imaging (MRI) is the ideal technique for assessing thoracic disc problems, since it is both non-invasive and very sensitive (7-9).

There is currently very little data about TDD in elderly people. Although symptomatic degenerative disc herniation occurs much less frequently in the thoracic spine than in other areas of the spine, the lack of research on the topic makes accurate diagnosis challenging (1). Moreover, there are few studies on pathology-related imaging of the thoracic spine (10-12). Therefore, the current study investigates the frequency of thoracic disc bulging/herniation in a group of elderly patients and seeks to determine the most common clinical presentations of such pathologies.

\section{METHODS AND MATERIALS}

\section{Subjects}

The study was planned as a retrospective study aimed at describing and characterizing the frequency of thoracic degenerative disc pathologies in a geriatric population. Participants were patients who presented at a physical medicine and rehabilitation outpatient clinic with upper back pain for more than 12 weeks and who were referred to the radiology department between January 2015 and August 2019.

The inclusion criteria of the study were: age 60 years and older and underwent an MRI scan of the thoracic vertebra due to complaints related to disc degeneration or to dorsalgia with suspected pathologies in the thoracic region. Patients with a chronic metabolic disorder, an infection, a malignancy, a compression fracture, or a previous surgery in the thoracic region were excluded. Patients with prominent kyphosis and/or rotoscoliosis were excluded as well.

This study was approved by the local ethics committee. The study was conducted in accordance with Good Clinical Practice and the Helsinki Declaration. Written and verbal informed consent was obtained from all participants.

\section{Study Design}

A total of 171 patients were included in the study. Each patient's thoracic vertebrae were scanned, resulting in a total of 2052 scanned levels (12disc levels). All T1- and T2-weighted sagittal and T2-weighted axial images were obtained with 1.5 T (Tesla) scanners (Magnetom; Siemens, Erlangen, Germany) and Philips Best (Netherlands). The following parameters were used: Sagittal T1 W (TR: 615/560, TE: 11/12, FOV: 320/220, slice thickness: 4/4 mm); sagittal T2 W (TR:3000/2930, TE: 83/110, FOV: 320/220, slice thickness: 4/4 mm); and axial T2 W (TR: 4340/4200, TE:109/140, FOV:160/200, slice thickness: $4 / 4 \mathrm{~mm}$ ). These parameters were used for both MRI devices. A picture archiving and communication system was used (PACS, General Electric, 
Chicago, IL, USA). All MR images were assessed by the same radiologist, who has ten years of experience in spinal neuroradiology. Two evaluations were done for each patient at an interval of one month to ensure intraobserver reliability.

Pathologies were characterized using updated lumbar disc nomenclature (13). The following pathologies were observed: protrusion, herniation, sequestration, bulging and extrusion, degeneration of the disc and the endplate, and Schmorl nodes. Intervertebral disc degeneration was determined using the Pfirrmann classification system, in which Pfirrmann grades I and II indicate no intervertebral disc degeneration, while Pfirrmann grades III,
$\mathrm{IV}$, and $\mathrm{V}$ indicate intervertebral disc degeneration (14). Changes in the endplate were evaluated using Modic classification and classified as type I, II, or III (15).

\section{Statistical Analysis}

The data were analyzed using the Statistical Package for the Social Sciences version 17 (SPSS Inc., Chicago, IL, USA). A Shapiro-Wilk test was used to check normality. Continuous variables were presented as the mean, standard deviation, and median (minimum-maximum) with regard to normality of distribution. Categorical variables were presented as frequency (percentage).

Table 1. Imaging findings by thoracic level and age group.

\begin{tabular}{|c|c|c|c|c|c|c|c|c|c|}
\hline \multicolumn{9}{|l|}{ Age } & \multirow[t]{2}{*}{$\mathrm{H}:$} \\
\hline \multirow{2}{*}{ Disc Level } & \multicolumn{2}{|c|}{$60-69(n: 102)$} & \multicolumn{2}{|c|}{$70-79(n: 56)$} & \multicolumn{2}{|c|}{$>80 \quad(n: 13)$} & \multicolumn{2}{|c|}{$\begin{array}{l}\text { Total patients } \\
(n=171)\end{array}$} & \\
\hline & B & $\mathrm{H}$ & B & $\mathrm{H}$ & B & $\mathrm{H}$ & B & $\mathrm{H}$ & $\mathrm{B}+\mathrm{H}$ \\
\hline $\mathrm{T} 1-2$ & 0 & 1 & 1 & 2 & 2 & 0 & 3 & 3 & 0 \\
\hline $\mathrm{T} 2-3$ & 1 & 3 & 1 & 6 & 2 & 1 & 4 & 10 & 0 \\
\hline T3-4 & 3 & 4 & 2 & 10 & 1 & 1 & 6 & 15 & 0 \\
\hline T4-5 & 1 & 6 & 1 & 11 & 1 & 2 & 3 & 19 & 0 \\
\hline T5-6 & 1 & 6 & 1 & 9 & 1 & 2 & 3 & 17 & 0 \\
\hline T6-7 & 4 & 11 & 4 & 18 & 1 & 5 & 9 & 34 & 3 \\
\hline T7-8 & 5 & 11 & 3 & 18 & 1 & 6 & 9 & 35 & 3 \\
\hline Т8-9 & 9 & 13 & 7 & 14 & 4 & 3 & 20 & 30 & 4 \\
\hline T9-10 & 12 & 14 & 13 & 17 & 4 & 2 & 29 & 33 & 3 \\
\hline T10-11 & 23 & 21 & 18 & 25 & 5 & 4 & 46 & 50 & 7 \\
\hline $\mathrm{T} 11-12$ & 24 & 21 & 17 & 25 & 4 & 5 & 45 & 51 & 4 \\
\hline T12-L1 & 12 & 14 & 14 & 14 & 3 & 3 & 29 & 31 & 4 \\
\hline Total & 95 & 125 & 82 & 169 & 29 & 34 & 206 & 328 & 28 \\
\hline
\end{tabular}

$\mathrm{H}$ : Herniation; B: Bulging. 
Table 2. Disc pathologies identified via MRI by intervertebral disc levels.

\begin{tabular}{|c|c|c|c|c|c|c|c|}
\hline \multirow{2}{*}{ Disc Level } & \multicolumn{2}{|c|}{ Bulging } & \multicolumn{3}{|c|}{ Herniation } & \multicolumn{2}{|c|}{ Total level } \\
\hline & Bulging & $\begin{array}{l}\text { Asymmetric } \\
\text { bulging }\end{array}$ & Protrusion & Extrusion & Sequestration & $\mathbf{n}$ & $\%$ \\
\hline $\mathrm{T} 1-2$ & 2 & 1 & 3 & 0 & 0 & 6 & 1.12 \\
\hline T2-3 & 3 & 1 & 10 & 0 & 0 & 14 & 2.62 \\
\hline T3-4 & 5 & 1 & 15 & 0 & 0 & 21 & 3.93 \\
\hline T4-5 & 2 & 1 & 19 & 0 & 0 & 22 & 4.11 \\
\hline T5-6 & 2 & 1 & 17 & 0 & 0 & 20 & 3.74 \\
\hline T6-7 & 8 & 1 & 34 & 1 & 0 & 44 & 8.22 \\
\hline T7-8 & 9 & 0 & 35 & 0 & 0 & 44 & 8.22 \\
\hline T8-9 & 20 & 0 & 30 & 0 & 0 & 50 & 9.35 \\
\hline T9-10 & 28 & 1 & 33 & 0 & 0 & 62 & 11.59 \\
\hline T10-11 & 46 & 0 & 48 & 1 & 1 & 96 & 17.94 \\
\hline T11-12 & 45 & 0 & 51 & 0 & 0 & 96 & 17.94 \\
\hline T12-L1 & 29 & 0 & 30 & 1 & 0 & 60 & 11.21 \\
\hline Total & 199 & 7 & 326 & 3 & 0 & 535 & 100,00 \\
\hline
\end{tabular}

\section{RESULTS}

A total of 171 participants ( 99 females and 72 males) were included in the study. The mean age of participants was $68.2 \pm 6.9$ years. Of the 171 participants, 102 were aged 60 to 69 years $(59 \%, 65), 56$ were aged 70 to 79 years $(32 \%, 75)$, and 13 were over $80(7 \%, 60)$.

Disc bulging or herniation was observed in 534 (26\%) of the total 2052 intervertebral disc levels analyzed in this study. When evaluated separately, 206 (10\%) discs demonstrated bulging. Bulging was most common at T10-11 ( $n=46,2.2 \%)$, followed by T11-12 $(n=45,2.1 \%)$ and T9-10 $(n=29,1.4 \%)$. Disc herniation was present in 328 (15.9\%) discs, most commonly at T11-12 $(n=51,15.5 \%)$, followed by T10$11(n=50,2.4 \%)$ and T7-8 ( $n=35,1.7 \%)$. Bulging and herniation were least common at T1-2, with an incidence of only $0.1 \%(n=3)$. Table 1 shows the evaluation and frequency of disc pathologies at each thoracic intervertebral level for each age group. Higher age is related to higher grades of herniation at every level (Table 2).

In our study, 12 cases had single-level bulging and 19 had single-level herniation. Single-level bulging occurred most frequently at T10-11 $(n=3)$, T11-12 $(n=3)$, and T12-11 $(n=3)$. Single-level herniation occurred most frequently at T6-7 $(n=5)$, T7-8 $(n=3)$, and T10-11 $(n=3)$. A total of 194 cases had multilevel bulging; 309 cases displayed multilevel 
Table 3. Frequency of single- and multilevel disc pathologies.

\begin{tabular}{|c|c|c|c|c|c|}
\hline & $\begin{array}{l}\text { Single level } \\
\text { Bulging }\end{array}$ & $\begin{array}{l}\text { Single level } \\
\text { Herniation }\end{array}$ & $\begin{array}{c}\text { Multilevel } \\
\text { Bulging }\end{array}$ & $\begin{array}{l}\text { Multi-level } \\
\text { Herniation }\end{array}$ & $\begin{array}{c}\text { Multi-level } \\
\text { Bulging+Herniation }\end{array}$ \\
\hline T1-2 & 0 & 0 & 3 & 3 & 0 \\
\hline T2-3 & 0 & 1 & 4 & 9 & 0 \\
\hline T3-4 & 1 & 0 & 5 & 15 & 0 \\
\hline T4-5 & 0 & 2 & 3 & 17 & 0 \\
\hline T5-6 & 0 & 0 & 3 & 17 & 0 \\
\hline T6-7 & 0 & 5 & 9 & 29 & 3 \\
\hline T7-8 & 0 & 3 & 9 & 32 & 3 \\
\hline T8-9 & 1 & 2 & 19 & 28 & 4 \\
\hline T9-10 & 1 & 0 & 28 & 33 & 3 \\
\hline T10-11 & 3 & 3 & 43 & 47 & 7 \\
\hline $\mathrm{T} 11-12$ & 3 & 2 & 42 & 49 & 4 \\
\hline T12-I1 & 3 & 1 & 26 & 30 & 4 \\
\hline Total & 12 & 19 & 194 & 309 & 28 \\
\hline
\end{tabular}

herniation, and 28 cases presented with both. Multilevel bulging was most common at T10-11 $(n=43)$ and T11-12 $(n=42)$. Multilevel herniation was most common at T11-12( $n=49)$ and T10-11 $(n=47)$. Multilevel bulging and herniation occurred together most commonly at $710-11(n=7)$. The frequency distributions of single- and multilevel disc pathologies are shown in Table 3.

All participants in the study presented with back pain. Forty-six participants had signs of neural compression. Of these 46 patients, 28 had herniation at multiple levels as well as a narrowed canal. One of these patients had a sequestrated disc, three had herniation at one level, six had herniation at two levels, four had herniation at three levels, and four had bulging at multiple levels.
An evaluation of disc degeneration at the thoracic level indicated that no participants had Pfirrmann grade 1 or 2 degeneration, $267(13 \%, 01)$ had grade 3 degenerations, $1628(79 \%, 34)$ had grade 4 degenerations, and $140(6 \%, 82)$ had grade 5 disc degeneration (Table 4).

In this study, endplate degeneration was found at 125 levels (6.09\%), and Schmorl nodes were found at 439 levels (21.3\%). Schmorl nodes were most common at T9-10, T10-11, T8-9, and T11-12 (in order of frequency) (Table 5).

\section{DISCUSSION}

This study was a retrospective investigation of 2052 levels of the thoracic spine in 171 geriatric patients 
who underwent MRI examinations of the thoracic vertebrae. The most common findings were disc degeneration, bulging, and herniation. Disc herniation occurred most frequently at T11-12. Bulging was most frequent at T10-11. Endplate degeneration was present in $6.09 \%$ of the 2052 levels analyzed, and Schmorl nodes were identified in $21.3 \%$.

Most TDHs are diagnosed in the third to fifth decades of life (1). Some studies have reported different ages of diagnosis; for instance, Sarsılmaz et al. found that thoracic disc pathologies were most frequent in the second and fourth decades (12). In this study, we examined patients in the sixth to eighth decades of life, and we accepted dorsalgia as an indication for thoracic vertebra MRI. MRI was used because of its high accuracy in diagnosing degenerative disc pathologies (1).
There are relatively few studies of age-related changes in the thoracic intervertebral discs. A previous retrospective study found that the prevalence of abnormal findings in the annuli, nuclei, and disc margins increases with increasing age, especially in the mid- and lower thoracic discs. Those authors suggest that disc recovery is unlikely after disc desiccation and height loss have occurred (16). Another study suggested a similar progression and found that developing degenerative changes became visible in imaging studies after 11 weeks (17). These results explain the increasing prevalence of thoracic disc pathologies with age. Similarly, our findings support a significant incidence of thoracic disc pathologies in patients over 60 years of age, especially in light of the reports by most literature that thoracic pathologies are very rare.

Table 4. Number of cases with each Pfirrmann grade by thoracic disk level.

\begin{tabular}{|c|c|c|c|c|}
\hline & gr3 & gr4 & gr5 & Total \\
\hline T1-2 & 24 & 142 & 5 & 171 \\
\hline $\mathrm{T} 2-3$ & 23 & 142 & 6 & 171 \\
\hline T3-4 & 24 & 140 & 7 & 171 \\
\hline T4-5 & 21 & 142 & 8 & 171 \\
\hline T5-6 & 21 & 141 & 8 & 171 \\
\hline T6-7 & 21 & 133 & 15 & 171 \\
\hline T7-8 & 21 & 131 & 17 & 171 \\
\hline T8-9 & 22 & 130 & 16 & 171 \\
\hline T9-10 & 22 & 130 & 17 & 171 \\
\hline T10-11 & 22 & 132 & 14 & 171 \\
\hline T11-12 & 23 & 128 & 17 & 171 \\
\hline T12-I1 & 23 & 137 & 10 & 171 \\
\hline \multirow[t]{2}{*}{ Total } & 267 & 1628 & 140 & 2052 \\
\hline & $13.01 \%$ & $79.34 \%$ & $6.82 \%$ & \\
\hline
\end{tabular}


Table 5. Distribution of Schmorl nodes and endplate degeneration at all intervertebral levels.

\begin{tabular}{|c|c|c|c|}
\hline Disc level & Number of Schmorl nodes & Disc level & $\begin{array}{l}\text { Endplate degeneration (level } \\
\text { numbers) }\end{array}$ \\
\hline T1 inferior & 7 & \multirow{2}{*}{$\mathrm{T} 1-2$} & \multirow{2}{*}{1} \\
\hline T2 superior & 7 & & \\
\hline T2 inferior & 6 & \multirow{2}{*}{$\mathrm{T} 2-3$} & \multirow{2}{*}{2} \\
\hline T3 superior & 6 & & \\
\hline T3 inferior & 6 & \multirow{2}{*}{ T3-4 } & \multirow{2}{*}{1} \\
\hline T4 superior & 6 & & \\
\hline T4 inferior & 8 & \multirow{2}{*}{ T4-5 } & \multirow{2}{*}{3} \\
\hline T5 superior & 8 & & \\
\hline T5 inferior & 11 & \multirow{2}{*}{ T5-6 } & \multirow{2}{*}{3} \\
\hline T6 superior & 8 & & \\
\hline T6 inferior & 17 & \multirow{2}{*}{ T6-7 } & \multirow{2}{*}{14} \\
\hline T7 superior & 15 & & \\
\hline T7 inferior & 32 & \multirow{2}{*}{$\mathrm{T} 7-8$} & \multirow[t]{2}{*}{13} \\
\hline T8 superior & 22 & & \\
\hline T8 inferior & 36 & \multirow{2}{*}{ T8-9 } & \multirow{2}{*}{20} \\
\hline T9 superior & 26 & & \\
\hline T9 inferior & 37 & \multirow{2}{*}{ T9-10 } & \multirow{2}{*}{26} \\
\hline T10 superior & 30 & & \\
\hline T10 inferior & 38 & \multirow{2}{*}{$\mathrm{T} 10-11$} & \multirow{2}{*}{22} \\
\hline T11 superior & 29 & & \\
\hline T11 inferior & 31 & \multirow{2}{*}{$\mathrm{T} 11-12$} & \multirow{2}{*}{15} \\
\hline T12 superior & 24 & & \\
\hline T12 inferior & 19 & \multirow{2}{*}{ T12-L1 } & \multirow{2}{*}{5} \\
\hline L1 superior & 10 & & \\
\hline Total & 439 & & 125 \\
\hline
\end{tabular}


Previous studies have found that TDH is most common at the middle to lower segments of the thoracic vertebrae, with a ratio of about $75 \%$ (17, 18). Sarsılmaz et al. showed that two-thirds of disc bulging and herniation pathologies occur from T7-8 to T11-12. They also report that T6-9 is the most commonly affected level in patients under 50 , while in older age groups, the most commonly affected levels are T10-12. Thus, they suggest that age-related changes in the vertebral column and ligaments may cause alterations in the localization of thoracic disc pathologies (12). Our findings are similar; we found that disc bulging and herniation are most common at T10-11 and T11-12 in patients over 60.

Another noteworthy finding of our study was that $36.2 \%$ of the patients had multilevel herniation. This incidence is higher than previously reported (about $20 \%$ to $26 \%)(4,19,20)$. Sarsilmaz et al. also found that $21.3 \%$ of participants had herniation at more than one level -consistent with previous literature but lower than our results (12). However, previous studies also suggest that thoracic disc pathologies increase with age. Increasing age coincides with more serious Modic and Pfirrmann grades. There is a strong correlation between changes in the endplates and in the intervertebral discs, as evidenced by changes in the MRI. The greater the level of disc degeneration, the closer the relationship between the Pfirmann and Modic classification systems (21). Furthermore, we included patients with complaints suggesting thoracic disc degeneration, which may have disproportionally increased the number of patients with multilevel pathologies, as they are more likely to present at the hospital with complaints.

In our study, both Schmorl nodes and endplate degeneration were most common at the low thoracic intervertebral levels. These findings align with previous literature $(12,18)$. There are several theories that attempt to explain the formation of
Schmorl nodes and their relationships with degenerative changes in the thoracic spine; however, the most popular explanation suggests that they occur when the cartilaginous endplate of the vertebral body is disrupted due to changes in physical properties. The most popular description suggests that Schmorl nodes occur when the cartilaginous endplate of the vertebral body is disrupted by physical changes $(18,22)$.

The present study has some limitations. First, we only included patients with dorsalgia. Previous studies have found that patients with thoracic disc pathologies have atypical presentations, such as abdominal pain, isolated chest pain, and isolated thoracic spine tenderness. Second, this is a retrospective study, which does not allow an investigation of causal relationships. Therefore, the frequency and severity of cases reported in this study may be higher than that in the general population of patients over 60. However, there are very few studies on this topic, and better characterization of thoracic disc pathologies is needed.

\section{CONCLUSION}

It is a given that thoracic disk pathologies are rare. However, they are not as rare nor as insignificant as previously suggested. They are known to cause severe neurological deficits, but their atypical clinical features make diagnosis difficult. To our knowledge, the present study is the most comprehensive characterization of thoracic disc pathologies in elderly patients. The results of our study indicate an increase in the prevalence of thoracic disc pathologies in elderly patients. Bulging, herniation, endplate changes, and Schmorl nodes occur more frequently at the lower intervertebral levels. Herniation and/or bulging at multiple levels are also more common than previously reported. 


\section{REFERENCES}

1. Mcinerney J, Ball PA. The pathophysiology of thoracic disc disease. Neurosurgical focus 2000;9(4):1-8. (PMID: 16833239)

2. Arseni $C$, Nash F. Protrusion of thoracic intervertebral discs. Acta Neurochirurgica 1963;11(1):3-33. (PMID: 13794536)

3. Haley J, Perry J. Protrusions of intervertebral discs: study of their distribution, characteristics and effects on the nervous system. The American Journal of Surgery 1950;80(4):394-404. (PMID: 14771346)

4. Linscott MS, Heyborne R. Thoracic intervertebral disk herniation: a commonly missed diagnosis. The Journal of emergency medicine 2007;32(3):235-8. (PMID: 17394983)

5. Carson J, Gumpert J, Jefferson A. Diagnosis and treatment of thoracic intervertebral disc protrusions. Journal of Neurology, Neurosurgery \& Psychiatry 1971;34(1):68-77. (PMID: 5551695)

6. Arce CA, Dohrmann GJ. Thoracic disc herniation: improved diagnosis with computed tomographic scanning and a review of the literature. Surgical neurology 1985;23(4):356-61. (PMID: 3975822)

7. Wilke A, Wolf U, Lageard P, Griss P. Thoracic disc herniation: a diagnostic challenge. Manual Therapy 2000;5(3):181-4. (PMID: 11034889)

8. Vanichkachorn JS, Vaccaro AR. Thoracic disk disease: diagnosis and treatment. JAAOS-Journal of the American Academy of Orthopaedic Surgeons 2000;8(3):159-69. (PMID: 10874223)

9. Wood KB, Garvey TA, Gundry C, Heithoff KB. Magnetic resonance imaging of the thoracic spine. Evaluation of asymptomatic individuals. The Journal of bone and joint surgery American volume 1995;77(11):1631-8. (PMID: 7593072)

10. Henkelman RM, Watts JF, Kucharczyk W. High signal intensity in MR images of calcified brain tissue. Radiology 1991;179(1):199-206. (PMID: 1848714)

11. Kasch R, Mensel B, Schmidt F, et al. Percutaneous disc decompression with nucleoplasty-volumetry of the nucleus pulposus using ultrahigh-field MRI. PloS one 2012;7(7):e41497. (PMID: 22848512)
12. Sarsılmaz A, Yencilek E, Özelçi Ü, Güzelbey T, Apaydın M. The incidence and most common levels of thoracic degenerative disc pathologies. Turkish Journal of Physical Medicine \& Rehabilitation (25870823) 2018;64(2). (PMID: 31453506)

13. Fardon DF, Williams AL, Dohring EJ, Murtagh FR, Rothman SLG, Sze GK. Lumbar disc nomenclature: version 2.0: Recommendations of the combined task forces of the North American Spine Society, the American Society of Spine Radiology and the American Society of Neuroradiology. The Spine Journal 2014;14(11):2525-45. (PMID: 24768732)

14. Pfirrmann CW, Metzdorf A, Zanetti M, Hodler J, Boos N. Magnetic resonance classification of lumbar intervertebral disc degeneration. Spine (Phila Pa 1976) 2001;26(17):1873-8. (PMID: 11568697)

15. Modic MT, Masaryk TJ, Ross JS, Carter JR. Imaging of degenerative disk disease. Radiology 1988;168(1):177-86. (PMID: 3289089)

16. Goh S, Tan C, Price R, et al. Influence of age and gender on thoracic vertebral body shape and disc degeneration: an MR investigation of 169 cases. The Journal of Anatomy 2000;197(4):647-57. (PMID: 11197538)

17. Girard CJ, Schweitzer ME, Morrison WB, Parellada JA, Carrino J. Thoracic spine disc-related abnormalities: longitudinal MR imaging assessment. Skeletal radiology 2004;33(4):216-22. (PMID: 14991248)

18. Pfirrmann CW, Resnick D. Schmorl nodes of the thoracic and lumbar spine: radiographic-pathologic study of prevalence, characterization, and correlation with degenerative changes of 1,650 spinal levels in 100 cadavers. Radiology 2001;219(2):368-74. (PMID: 11323459)

19. Yue B, Chen B, Zou Y-w, et al. Thoracic intervertebral disc calcification and herniation in adults: a report of two cases. European Spine Journal 2016;25(1):11823. (PMID: 26329651)

20. Giblin EM, Hochheiser GM. Thoracic disk herniation resulting in acutely progressing paraplegia in a pediatric patient. Pediatric emergency care 2008;24(8):550-3. (PMID: 18708901) 
21. Rodrigues LMR, Yoshino CV, Costa AB. Lumbar alterations in magnetic resonance: correlation between Modic and Pfirmann classifications. Coluna/Columna 2014;13(3):202-5. (DOI: 10.1590/ s1808-18512014130300263)

22. Schmorl G. Über knorpelknötchen an den wirbelbandscheiben. Rofo Fortschr Geb Rontgenstr Neuen Bildgeb Verfahr 1928;38:265-79.
24. Bilgic S, Acaroglu R. Effects of listening to music on the comfort of chemotherapy patients. West J Nurs Res 2017: 39(6): 745-62. ( PMID: 27515501).

25. Umbrello M, Sorrenti T, Mistraletti G, Formenti $P$, Chiumello D, Terzoni S. Music therapy reduces stress and anxiety in critically ill patients: a systematic review of randomized clinical trials. Minerva anestesiologica, 2019; 85(8): 886-98. (PMID: 30947484). 